\title{
Septic arthritis caused by treatment resistant Pseudomonas cepacia
}

\author{
Eric L Matteson, W Joseph McCune
}

\begin{abstract}
A case of septic arthritis of the knee caused by Pseudomonas cepacia following an intraarticular corticosteroid injection in a patient with a history of osteoarthritis is presented. This is the second report of this agent causing infection in a diarthrodial joint, which proved difficult to eradicate despite in vitro antibiotic sensitivity.
\end{abstract}

About $5-10 \%$ of all cases of septic arthritis occurring in adults are caused by Gram negative bacilli. ${ }^{12}$ The two most important factors predisposing to the development of Gram negative arthritis are intravenous drug use and an impaired host defence. ${ }^{13}$

Pseudomonas cepacia, an organism traditionally regarded as having low pathogenicity in humans, has become recognised as an important nosocomial pathogen. ${ }^{45}$ Two reports of $P$ cepacia arthritis treated successfully on the basis of in vitro antibiotic sensitivities have appeared. ${ }^{67}$ In one of these septic arthritis of the ankle followed repeated intra-articular steroid injection from a contaminated vial of methylprednisolone. ${ }^{7} \mathrm{We}$ report a case of $P$ cepacia causing septic arthritis of the knee that remained culture positive for 44 days despite intravenous antibiotics, repeated needle aspiration of synovial fluid, and open synovectomy.

\section{Case report}

A 72 year old woman with a history of osteoarthritis was admitted for evaluation of severe right knee pain, swelling, and fever. One week before admission she had received an intraarticular corticosteroid injection into her right knee.

The past medical history was unremarkable except for allergy to penicillin. Physical examination showed a pleasant, disoriented woman. Her temperature was $37.6^{\circ} \mathrm{C}$, blood pressure $150 / 94 \mathrm{mmHg}$, pulse $104 /$ minute, and respiratory rate $20 /$ minute. Positive findings included marked warmth, tenderness, and swelling of the right knee. A stool specimen was positive for occult blood. An $x$ ray examination of the knees showed changes of moderately severe osteoarthritis with joint space narrowing and osteophyte formation.

Treatment with vancomycin and tobramycin was started. After 72 hours Gram negative rod isolates were transferred from chocolate blood agar medium to MacConkey medium and found to be lactose and oxidose negative. $P$ cepacia was identified using the criteria outlined by
Antimicrobial susceptibility of $P$ cepacia isolates

\begin{tabular}{lll}
\hline Antimicrobial agent & Sensitivity $^{*}$ & $M I C^{*}(\mu \mathrm{g} / \mathrm{ml})$ \\
\hline Amikacin & $\mathrm{R}$ & $>32 \cdot 00$ \\
Ampicillin & $\mathrm{R}$ & $>16.00$ \\
Cephazolin & $\mathrm{R}$ & $>32.00$ \\
Cephamandole & $\mathrm{R}$ & $>32.00$ \\
Cefoxitin & $\mathrm{R}$ & $>32.00$ \\
Chloramphenicol & $\mathrm{R}$ & 16.00 \\
Gentamicin & $\mathrm{R}$ & $>16.00$ \\
Piperacillin & $\mathrm{S}$ & 16.00 \\
Ticarcillin & $\mathrm{R}$ & $>128.00$ \\
Trimethoprim/ & & $<0.50 / 9.50$ \\
Sulphamethoxazole & $\mathrm{S}$ & $>16.00$ \\
Tobramycin & $\mathrm{R}$ & 8.00 \\
Imipenem & $\mathrm{I}$ & $>128.00$ \\
Ticarcillin & $\mathrm{R}$ & 16.00 \\
Ceftriaxone & $\mathrm{S}$ & $<4.00$ \\
Ceftazidime & $\mathrm{S}$ & \\
\hline
\end{tabular}

${ }^{*} \mathrm{~S}=$ sensitive; $\mathrm{R}=$ resistant $\quad \mathrm{I}=$ intermediate; $\mathrm{MIC}=$ minimum inhibitory concentration.

Otto and Pickett ${ }^{8}$ as modified for the APIc rapid non-fermenting technique strips (Analytab Products, Plainview, NY). ${ }^{9}$ Blood and spectum cultures remained negative. Antimicrobial minimum inhibitory concentrations were determined by the microtitre dilution technique (table).

The antibiotic was changed to trimethoprimsulphamethoxazole. Synovial fluid cultures from the right knee remained positive for $P$ cepacia. Eight days after admission the patient underwent open synovectomy. After 13 days trimethoprim-sulphamethoxazole was discontinued because of drug rash and renewed fever. Intravenous chloramphenicol was begun. After initial improvement, fever and effusion recurred. Intravenous ceftazidime was started. On the 36th day repeat open drainage and synovectomy were performed. The patient was then transferred to this institution. Repeat synovial cultures showed $P$ cepacia with an identical antimicrobial susceptibility profile. Intravenous ceftazidime ( $2 \mathrm{~g}$ every eight hours) was continued; serum peak concentrations were $190 \mu \mathrm{g} / \mathrm{ml}$ and bactericidal titres were $>1 / 256$. Daily aspiration of synovial fluid was performed with a large bore needle. The synovial fluid white blood cell count gradually decreased to less than $1.25 \times 10^{9}$ cells/ 1 . Cultures became negative after eight days. Subsequent $x$ rays of the right knee showed progressive joint space narrowing and confluent subchondral bone plate erosions. During this period the patient underwent colectomy to control recurrent severe colonic bleeding due to arteriovenous malformation. The knee pain slowly resolved and the patient's nutritional status improved. After 60 days antibiotics were discontinued. 


\section{Discussion}

Pseudomonas cepacia is ubiquitous in the environment and has been isolated from many human sources as well as aqueous solutions and equipment found in hospital. ${ }^{40-13}$

Treatment of $P$ cepacia is often difficult. It is reported to have in vitro sensitivity to trimethoprim-sulphamethoxazole,${ }^{14}$ chloramphenicol, ${ }^{15}$ and cefoperazone. ${ }^{6}$ It is generally resistant to polymyxins, aminoglycosides, first and second generation cephalosporins, and traditional antipseudomonal penicillins such as ticarcillin. ${ }^{10}$ In marked contrast with the previously cited report in which the $P$ cepacia organism was found to be sensitive togentamicin, ${ }^{7}$ the organism recovered was more typical. It was not only gentamicin resistant, but also proved difficult to eradicate in vivo despite the use of third generation cephalosporins to which in vivo sensitivity was shown.

Doctors evaluating otherwise healthy patients presenting with septic arthritis following open trauma, surgery, or joint injections must be alert to the possibility of such unusual organisms causing infection. Even with the available antibiotic armamentarium, treatment will continue to pose a major challenge.

1 Goldenberg D L, Cohen A S. Arthritis due to gram-negative bacilli. Clin Rheum Dis 1978; 4: 197-208.
2 Schmid F. Bacterial arthritis. In: McCarty D S, ed. Arthritis and allied conditions. Philadelphia: Lea and Febiger, 1989: and allied

3 Gifford D B, Patzakis M, Luller D, Swezy L. Septic arthritis due to pseudomonas in heroin addicts. $\mathcal{F}$ Bone foint Surg [Am] 1975; 57: 631-5.

4 Conly J M, Klass L, Lerson L, Kennedy J, Low D E Harding G K M. Pseudomonas cepacia colonization and infection in intensive care units. Can Med Assoc F 1986; 134: 363-6.

5 Ederer G M, Matsen J M. Colonization and infection with Pseudomonas cepacia. F Infect Dis 1972; 125: 613-8.

6 Smith M A, Trowers N R H, Klein R S. Cervical osteomyelitis caused by Pseudomonas cepacia in an intravenous drug caused by Pseudomonas cepacia in an in

7 Kothari T, Reyes M P, Brooks N, Brown W J, Lerner A M Pseudomonas cepacia septic arthritis due to intraarticular injections of methylprednisolone. Can Med Assoc f 1977; 116: $1230-5$

8 Lennette E H, Spaulding E H, Trauant J P. Manual of clinical microbiology. Washington DC: American Society for Microbiology, 1980 .

9 Otto L A, Pickett M J. Rapid method for indentification of gram negative, nonfermentative bacilli. $\mathcal{f}$ Clin Microbiol 1976; 3: 566-75.

10 Goldman D A, Klinger J D. Pseudomonas cepacia: biology, mechanisms of virulence, epidemiology. 7 Pediatr 1986; 108: 806-12.

11 Craven D E, Moody B, Connolly M G. Pseudomonas cepacia. N Engl f Med 1981; 305: 621-3.

12 Gilardi G L. Pseudomonas cepacia: culture and laboratory identification. Laboratory Management 1983; 21 : 29-32.

13 Weinstein R A, Smori T G, Anderson R L, et al. Pressure transducers as a source of bacteremia after open heart surgery: report of an outbreak and guidelines for prevention. Surgery: report of an out

14 Moody M R, Young V M. In vitro susceptibility of Pseudomons cepacia and Pseudomonas maltophilia to trimethoprim and trimethoprim-sulfamethoxazole. Antimicrob Agents Chemother 1975; 7: 836-9.

15 Pallent L J, Hugh W B, Grant D J W. Pseudomonas cepacia as contaminant and infective agent. $\mathcal{F}$ Hosp Infect 1983; 4: 9-13. 